\title{
L'exploitation agricole, entre famille et entreprise : 60 ans de débats et d'itinéraire de recherche personnel
}

Jean Pluvinage ${ }^{1}$

\section{Introduction et problématique générale de cette réflexion rétroactive}

Pourquoi revenir sur un débat récurrent, dont les termes ont été largement posés tout au long du $\mathrm{XX}^{\mathrm{e}}$ siècle ? Tout simplement parce qu'au-delà de choix idéologiques vigoureux des parties prenantes à ce débat sur l'agriculture familiale (organisations sociales et syndicales agricoles, pouvoirs publics, enseignants et chercheurs qui se sont investis dans ce débat), la question reste contingente de l'évolution de l'agriculture elle-même, de son poids économique, de son cadre institutionnel, qui rouvre une controverse que l'on croyait close, en Europe, depuis la mise en place de la $\mathrm{PAC}^{2}$.

Il est utile de repositionner ce débat exploitation agricole familiale (EAF) vs entreprise agricole (EA), parallèlement à celui de l'évolution de l'agriculture et de la politique agricole, en écho à des travaux de sociologie plus récents s'interrogeant sur le développement de l'agriculture de firme, voire d'un capitalisme financier agricole (Hervieu et Purseigle, 2011), ou ceux d'économistes sur l'évolution du modèle européen de la moyenne exploitation (Neveu, 2010).

Le débat entre les avantages et les inconvénients de la grande entreprise en agriculture, qualifiée de "capitaliste " en référence aux économistes néoclassiques, par opposition à l'exploitation agricole paysanne (Tchayanov, 1990), a été un débat fondateur tant des controverses professionnelles que celles d'économistes et d'agronomes, observateurs des processus de modernisation et de transformation du secteur agricole. Bien évidemment, les différentes organisations syndicales agricoles

1. Ancien directeur de recherches à l'INRA, département Sad, 7 passage du chemin vert, 75011 Paris, France, jpluvinage@gmail.com

2. En toile de fond, j’adopte ici pleinement les interprétations de C. Laurent et J. Rémy (2000), qui ont décrit la genèse du concept de l'exploitation agricole comme outil statistique et catégorie politique, en mettant en avant l'historicité de cette catégorie.

Ce chapitre de l'ouvrage L'agriculture en famille : travailler, réinventer, transmettre est publié en Open Access sous licence creative commons CC-BY-NC-ND permettant l'utilisation non commerciale, la distribution, la reproduction du texte, sur n'importe quel support, à condition de citer la source. 
remobilisent un tel débat, en fonction de leur base sociale et des objectifs d'intervention publique attendus, sur la compétitivité de l'agriculture exportatrice et l'emploi agroalimentaire d'un côté, sur l'emploi agricole, l'alimentation localisée et l'environnement de l'autre. Remarquons aussi qu'un discours se voulant souvent consensuel sur l'EAF, pour des raisons d'hégémonie syndicale, a souvent masqué une lecture plus contrastée de la caractérisation de la réalité économique, face aux évènements de l'actualité.

Aujourd'hui, la notion d'entreprise qui se substitue, implicitement ou explicitement, à celle d'exploitation est clairement remobilisée dans le discours à la FNSEA (Fédération nationale des syndicats d'exploitants agricoles), comme au JA (Jeunes Agriculteurs), comme fondement de la viabilité de l'activité agricole dans l'avenir, par opposition aux positions paysannes de la Confédération paysanne, du $\mathrm{MODEF}^{3}$, voire de la coordination rurale. Si l'épithète « familiale » de l'exploitation reste largement revendiquée, tout au moins dans une certaine mesure, par toutes les parties prenantes, l'équilibre réel de la balance EAF/EA mérite d'être revisité.

C'est pour moi l'occasion de rendre compte, dans ce chapitre, d'une mise en perspective de travaux menés sur l'exploitation agricole durant la période 1950-2010, qu'il s'agisse de mes propres travaux (à partir des années 1970), ou de ceux d'économistes enseignants et chercheurs qui se sont interrogés, durant cette période, depuis les années 1950 sur la réalité socio-économique de l'évolution des exploitations agricoles, et sur la pertinence de différents schémas explicatifs de ces évolutions. Cette rétrospective consiste plus en un témoignage fondé sur une trajectoire de plus de quarante ans de vie professionnelle, ouvrant une réflexion et une discussion sur les interrelations entre une histoire de l'économie rurale française et l'objet exploitation agricole, qu'une recension exhaustive de la littérature scientifique produite sur l'EAF.

Cette controverse EAF/EA avec salariés, se décline dans des termes différents depuis les années 1950, selon trois périodes principales :

1) de 1950 aux années 1960-1970. ${ }^{4}$

Après la seconde guerre mondiale, ce débat sur les limites de l'exploitation agricole familiale, par rapport à l'entreprise avec salariés a été très présent, y compris en grande culture. Bernard Poulain, agriculteur du Bassin parisien, créateur du premier CETA (Centre d'expérimentation des techniques agricoles), défend la nécessité d'entreprises agricoles diversifiées de grande taille économique avec suffisamment de salariés pour bénéficier des avantages de la spécialisation et du plein emploi des machines, et une conception rénovée de l'entreprise et des rapports aux salariés fondée sur les principes du catholicisme social. Il s'agissait aussi d'une réaction à

3. Mouvement d'organisation et de défense des exploitations familiales.

4. Le choix d'un créneau de dates large, 1960-1970, tient au fait que cette sous-période est réellement une transition entre le choc des premières manifestations de 1960 et la première loi d'orientation agricole, puis celle de 1962, la loi sur l'élevage de 1966 et la mise en place des différents outils d'appui au développement et à l'intervention sur les structures (SUAD, SAFER, CDOA, etc.) 
l'agrarisme traditionnel des organisations agricoles plus soucieuses de leur protection économique que d'une remise en cause de l'organisation de la production agricole appelée par les forces montantes de la JAC (Jeunesses agricoles chrétiennes). Les mots-clés des pouvoirs publics sont : augmentation quantitative de la production, modernisation et productivité ;

2) des années 1960-1970 à la première réforme de la PAC de 1992.

À une époque où le développement industriel et général en France est rapide, une décision fondamentale est prise par l'État (puis relayée par la Communauté économique européenne à partir de 1970), celle d'adapter radicalement les structures de l'agriculture à son époque, par un agrandissement régulé des exploitations, par la mise en place d'une organisation des marchés et des filières, où les agriculteurs sont censés être mieux défendus s'ils acceptent des règles d'organisation importantes. C'est l'époque des groupements de producteurs, de la généralisation des offices, et des comités économiques pouvant contraindre, dans certaines conditions, l'ensemble des producteurs à respecter certaines règles de commercialisation, mais aussi celle de l'encouragement prodigué aux CUMA (Coopératives d'utilisation du matériel agricole) pour accéder à la mécanisation.

En même temps, à partir des lois d'orientation agricole de 1960 et 1962, les pouvoirs publics interviennent directement dans la structuration des exploitations agricoles et pas seulement sur le parcellaire, comme c'était le cas jusque-là dans les opérations de remembrement rural.

Il s'agit d'inciter le maximum d'exploitations, quand elles le peuvent, à se rapprocher d'un modèle jugé techniquement, socialement et politiquement idéal, celui de « l'exploitation agricole à 2 UTH (unité de travail humain, mesure d'équivalent annuel) ", la deuxième UTH étant supposée de nature majoritairement familiale, minoritairement salariée ;

3) depuis la première réforme de la PAC, en 1992, jusqu’à la période actuelle.

On entre progressivement dans une période où le découplage des financements publics de la production agricole va s'imposer, tant pour des raisons de limites budgétaires, que pour des raisons de mise en conformité avec les règles de l'Organisation mondiale du commerce, et enfin aussi pour des raisons de gestion durable de l'environnement. On verse de moins en moins de subventions au soutien des marchés, les prix de ventes des produits devant s'aligner sur les cours mondiaux, et de plus en plus de subventions à l'hectare, ce qui encourage directement l'agrandissement. L'accroissement de la taille du matériel de culture, d'abord réalisée pour effectuer les opérations culturales dans de meilleures conditions, est de fait un puissant aiguillon à accroître sa superficie dès que l'on trouve des terres disponibles. Autrement dit, si la production maximale à l'hectare n'est plus le mot-clé, la productivité du travail, comme dans le reste de l'économie française, reste le principal mot d'ordre des pouvoirs publics et des organisations syndicales majoritaires.

Concrètement, l'accroissement de la taille des exploitations, et donc la disparition des petites structures, devient la principale locomotive de l'accroissement du revenu, 
dans les exploitations qui se consacrent à la production de biens alimentaires de base $^{5}$.

Simultanément, un certain nombre d'innovations techniques reposent la question de l'organisation du travail dans les productions animales. Par exemple en élevage laitier, de plus grandes réticences aux astreintes biquotidiennes de la traite, la spécialisation des régions et celle des exploitations, aboutissent à la création de troupeaux plus importants et un petit développement du salariat dans des exploitations d'élevage, avec parfois la mise en commun en un seul troupeau et un seul bâtiment des animaux de plusieurs fermes, qui génère une renaissance du salariat en élevage.

L'hypothèse centrale que nous interrogeons tout au long de ce chapitre et sur laquelle nous reviendrons en conclusion avec une interrogation en termes de perspectives, c'est que le modèle d'EAF " professionnelle » phare de la période des années 19601992, est de plus en plus soumis à de fortes tensions, par rapport à une industrialisation aujourd'hui renouvelée de l'agriculture, même si celle-ci est en partie remise en cause par différents acteurs.

\section{Des années 1950 jusqu'aux années 1960-1970: la mise en place des outils de la modernisation de l'exploitation agricole}

\section{Le contexte général : produire plus et moderniser toute I'agriculture, avec comme objectif l'obtention d'un revenu et de conditions de vie équivalentes à celles des urbains}

De la fin de la seconde guerre mondiale aux années 1960, il faut produire plus, à tout prix, et profiter d'une mécanisation qui semble s'adapter dans un premier temps à l'éclatement des structures d'exploitations agricoles françaises, par une miniaturisation d'un machinisme agricole initialement conçu pour les fermes américaines. Il s'agit plus en fait de remplacer la traction animale par de la traction mécanique pour produire plus de biens alimentaires, pour un pays et un continent sortant de dix ans de pénurie, que d'accroître globalement la productivité du travail, y compris dans les grandes exploitations de certaines zones, comme le Bassin parisien qui vont se spécialiser en céréaliculture, et cultures industrielles, en abandonnant l'élevage laitier, traditionnel dans ces grandes fermes. Cette spécialisation en productions

5. Il faut nuancer notre affirmation quand on aborde les exploitations qui se consacrent aux productions de qualité, qui peuvent bénéficier dans certaines conditions de marché, de prix et de revenus améliorés, leurs productions étant protégées par des certifications officielles (agriculture biologique $[\mathrm{AB}]$, label rouge, appellation d'origine contrôlée $[\mathrm{AOC}]$, appellation d'origine protégée $[\mathrm{AOP}]$ et indication géographique protégée [IGP]), de même que pour les exploitations développant des activités de ventes directes de produits alimentaires ou de services dans des logiques de proximité locale ou régionale. 
végétales de grandes exploitations s'appuie sur une intensification rendue possible par une industrie de l'azote pas cher, des variétés végétales adaptées, et un début d'utilisation des traitements phytosanitaires.

Paradoxalement, à mesure que cette grande culture s'industrialise, elle devient de plus en plus familiale, diminuant radicalement les salariés sur les fermes, d'abord les charretiers et les vachers, puis aussi les conducteurs de tracteurs à mesure que ceuxci accroissent leur puissance et que les moissonneuses batteuses effectuent d'un seul coup les opérations de récolte pour l'élaboration d'un produit fini directement livré à la coopérative et non plus stocké à la ferme.

Parallèlement, les exploitations d'élevage évoluent beaucoup moins vite, handicapées par de faibles potentialités d'intensification du travail, que ce soit dans la récolte du fourrage ou des soins apportés aux animaux.

Notons aussi que le développement des CUMA, d'abord créées pour permettre d'accéder dans de meilleures conditions à un matériel rare et coûteux, est aussi une réponse appropriée à l'adaptation nécessaire de l'exploitation à la taille de machines dont l'optimum d'utilisation est adapté à des exploitations de grande taille.

La question centrale est alors de produire plus en assurant aux agriculteurs un revenu "décent ». La " parité des revenus ", point d'entrée de la revendication des agriculteurs, est un mot-clé du syndicalisme agricole demandant à l'État d'intervenir sur tout ce qui peut influencer les prix des produits agricoles ou des intrants ${ }^{6}$. En même temps, on développe une politique industrielle de production d'azote pas cher, et on attribue des subventions à l'acquisition de matériel agricole. La politique constante des pouvoirs publics sera d'accélérer la modernisation de l'agriculture, initiée par la mécanisation après la seconde guerre mondiale qui sera rapidement généralisée : 1,2 millions de tracteurs en 1970, contre 200000 en 1955, accompagnent l'intensification générale de la production agricole, conduisant à un doublement du volume produit en 20 ans. Cependant dans le même temps, un tiers des exploitations a disparu, et l'emploi agricole qui représentait $30 \%$ de l'emploi total en 1955, n'en représente plus que $15 \%$ en 1970 .

\section{Position des économistes sur les conséquences de l'industrialisation de l'agriculture sur les différentes formes d'exploitations agricoles}

Pour beaucoup de chercheurs ou d'enseignants ayant commencé leur activité professionnelle dans les années 1960, le questionnement de la modernisation de

6. Les grandes discussions État/Syndicats agricoles reposent sur la comparaison des indices de prix entre celui des prix des produits agricoles (IPAP : indice des prix agricoles à la production) et celui des produits industriels (IPPINEA : indice des prix des produits industriels nécessaires aux exploitations agricoles). Très clairement, le rôle de l'État est d'éviter que se referme le " ciseau " entre les prix agricoles et les prix des produits industriels ; ceci est conforme avec le mot d'ordre de l'industrialisation de l'agriculture. 
l'agriculture tel qu'il est posé par R. Dumont, avec un triple regard (d'agronome, d'économiste, et d'une certaine manière un regard de sociologue sur la paysannerie) est assez représentatif du débat de cette époque.

La question du développement de la productivité par la mécanisation est une obsession chez R. Dumont quand, dans les années 1950, il compare l'agriculture des Hautes-Alpes à celle des plaines américaines ${ }^{7}$ : un quintal de foin par journée de travail à Saint-Chaffrey, contre dix tonnes aux USA, soit un écart de productivité de 1 à 100.

Si je situe ce débat, du début de ma carrière à l'Institut national agronomique ParisGrignon, des courants comme ceux de la chaire d'économie rurale de Grignon, dirigée par J. Chombart de Lauwe et J. Poitevin, et ceux de la chaire d'agriculture comparée animée par R. Dumont et M. Mazoyer, s'intéressent à l'économie de l'exploitation agricole dans deux perspectives très différentes.

Pour J. Chombart de Lauwe et al. (1963), l'objectif est de promouvoir l'industrialisation de l'agriculture, c'est-à-dire appliquer à l'exploitation agricole les règles d'organisation et de gestion propre à l'industrie, en commençant par la comptabilité ${ }^{8}$ adaptée à partir du plan comptable général ${ }^{9}$. Contrairement à $\mathrm{R}$. Dumont, il ne préconise pas de réforme des structures d'exploitation comme préalable à la modernisation, mais de la formation pour produire et sélectionner de bons gestionnaires d'exploitation. Pour lui, l'augmentation permanente du capital d'exploitation et sa gestion raisonnée est le seul moyen d'assurer l'avenir de l'exploitation, et la sélection de celles qui devront perdurer. Il prône pour les exploitations l'obtention d'un profit d'entreprise, avant de considérer l'angle du revenu de l'agriculteur. Dans un esprit de contribution à la modernisation, il contribue largement à la multiplication des centres de gestion dans des contextes agricoles régionaux variés, par ailleurs excellents débouchés pour les ingénieurs formés à Grignon.

7. La lecture des voyages d'un agronome en France (Dumont, 1951) est presque brutale sur cette question : "Il ne peut y avoir en polyculture classique céréales fourrages une productivité suffisante du travail pour une famille équipée, si l'on descend en dessous de 30 hectares. Ce minimum... se situe très loin de l'optimum nécessaire à l'emploi du matériel moderne de culture, qui exige plusieurs centaines d'hectares, et en demandera demain plusieurs milliers, comme en URSS » dans la conclusion générale intitulée notre agriculture en péril (p. 460).

8. J. Chombart de Lauwe et al. (1963), dans l'introduction de leur ouvrage sur la nouvelle gestion des exploitations agricoles proposent une définition : "l'exploitation agricole est une unité économique dans laquelle l'agriculteur pratique un système de production en vue d'augmenter son profit ", ce qui est une référence implicite à la plaine de Versailles, région de grande culture, ou à l'Eure, département où il appuie la création du premier centre de gestion en France en 1954. La question essentielle de ces exploitations de grande culture (polyculture élevage, à l'époque) est la mesure de la « rentabilité » du capital propre mobilisé dans les exploitations.

9. Le plan comptable général, réactualisé périodiquement depuis sa création en 1941, a pour objet de rendre comparable les résultats économiques et les bilans à des fins d'information des actionnaires et de gestion financière, et aussi fiscales. 
Très clairement, dans ce débat, mes travaux se situent plus dans le domaine de l'agriculture comparée et du développement, à cause de son champ large d'investigations (technique, économique, social et comparatif), de ses méthodes d'approche des exploitations par enquêtes et observations des pratiques agricoles de gestion $\mathrm{du}$ système de production, dans des cadres d'analyse mieux adaptés à la réalité de la majorité des exploitations agricoles.

Ceci n'est pas étranger à ma rencontre ultérieure dans les années 1990 avec le département Sad (Systèmes agraires et développement, devenu par la suite département des Sciences pour l'action et le développement) de l'Inra.

\section{Des années 1960-1970 à 1992 : le triomphe de la moyenne exploitation agricole}

\section{L'encadrement institutionnel des exploitations familiales moyennes : l'exploitation agricole à 2 UTH devient la référence}

À partir des années 1960, corrélativement à l'ouverture du marché européen à l'ensemble des productions agricoles, construit d'abord pour les céréaliers et les grandes cultures, la question de la modernisation de l'ensemble de l'agriculture va se poser dans d'autres termes. L'avènement de la $\mathrm{V}^{\mathrm{e}}$ république est une période d'expansion économique sans précédent sur le plan industriel avec des politiques incitatives et une planification nationale qui n'affiche plus comme objectif la reconstruction mais un développement économique renouvelé. L'agriculture n'échappe pas à ces grands bouleversements, sous la pression des agriculteurs qui manifestent pour la "parité de leurs revenus ", en réclamant des prix rémunérateurs, puis par l'arrivée au pouvoir syndical de jeunes agriculteurs comprenant bien, en référence à l'agriculture néerlandaise, allemande et danoise, que ceci n'est pas possible sans une politique publique de modernisation des structures, complétant les politiques de soutien des marchés.

C'est ainsi que dans les lois d'orientation de 1960 et 1962, va naître le modèle de l'exploitation agricole à 2 UTH. Il représente de fait une sorte d'archétype, conforme à beaucoup d'exploitations réelles, mais aussi un objectif de structure prescrit aux petites exploitations pour obtenir un revenu suffisant, devenir des exploitations professionnelles ${ }^{10}$ et perdurer en tant que structure de production dans l'avenir. En échange, les pouvoirs publics s'engagent dans une politique de "soutien différencié des structures agricoles " quasiment inexistante auparavant, ou les crédits étaient essentiellement consacrés à l'aménagement rural (électrification, adduction d'eau, remembrement) ou aux grands aménagements régionaux, menés par des sociétés

10. Ce terme sera repris ultérieurement par les statisticiens agricoles, pour qualifier les exploitations de plus de 0,75 personne à temps plein, obtenant une marge brute de plus de 9600 euros, soit l'équivalent de ce que l'on obtient sur 12 hectares de blé. 
d'économie mixtes. D'une certaine manière, ce sont les leçons de R. Dumont des années 1950, prônant des réformes de structures des exploitations pour appliquer la modernisation, qui sont mises en œuvre 15 ans plus tard par E. Pisani, ministre de l'Agriculture de 1960 à 1966.

Ce modèle est familial dans son expression la plus fréquente, tout en acceptant un certain flou sur le statut du deuxième travailleur : conjoint de l'exploitant, aide familiale, salarié agricole.

Le modèle de référence néerlandais qui a si bien réussi la modernisation de son élevage laitier sur des superficies agricoles par exploitation inférieures à celle des exploitations d'élevage françaises fascine par l'ampleur des progrès économiques obtenus ; du coup il donne l'apparence d'être accessible à presque tous les agriculteurs français. Bien des régions agricoles vont adopter cette stratégie de développement, la Bretagne en étant un des exemples les plus aboutis.

Ainsi, le débat sur les avantages et inconvénients de l'EA avec des salariés agricoles, par rapport à l'EAF, devient secondaire par rapport à celui des conditions de l'adoption de l'intensification de la production. La logique de l'industrialisation semble l'emporter sur la logique de l'agrandissement ; en quelque sorte, tout le monde fait semblant de penser que le mécanisme d'exclusion lié à l'agrandissement des exploitations sur un espace limité, peut être contourné par un développement intense du capital d'exploitation par unité de superficie, qui compenserait les inconvénients du non passage à la grande entreprise.

Ceci est renforcé par deux tendances convergentes : 1) l'EAF est riche d'une maind'œuvre plus ou moins rémunérée qui offre une grande souplesse de gestion $\mathrm{du}$ système productif à condition qu'on la motive par des conditions de travail modernisées (rôle du machinisme) et une reconnaissance professionnelle avec des diplômes équivalents en niveau à ceux des autres métiers et des conditions de vie plus décentes que celles héritées de la France de la III et $\mathrm{IV}^{\mathrm{e}}$ république ; 2) le coût du salariat agricole augmente dans des proportions importantes, d'abord par ce que les lois de protection sociale s'étendent plus efficacement qu'auparavant, et ensuite par ce que le montant du salaire minimum agricole est aligné sur le SMIC en 1968, ce qui équivaut à une augmentation de $33 \%$. Ceci repose à l'époque la question de l'avenir du salariat en agriculture, conduisant à une relance de la course à la croissance de la taille du matériel.

Dès la fin des années 1960, l'impact de la PAC a été suffisant pour saturer les marchés européens (céréales et poudre de lait) et s’impose alors la nécessité de produire dans des conditions de marché plus difficiles, où les exploitations agricoles ne pourront voir leur revenu progresser que si elles s'agrandissent ou modernisent leur appareil de production.

C'est le choix qui est proposé par le commissaire européen à l'agriculture, S. Mansholt : être plus sélectif dans les aides à la modernisation distribuées en encourageant en même temps un certain nombre d'agriculteurs à quitter l'agriculture pour que les autres puissent s'agrandir. 
Les " plans de développement des exploitations ${ }^{11}$ prétendent résoudre, une fois pour toutes, la question des disparités en ciblant l'effort de modernisation sur les exploitations agricoles de taille intermédiaire. Ces dernières sont soutenues à condition que les exploitants prennent des engagements importants en investissements et souscrivent à des objectifs de revenus " de parité " à atteindre au bout de six années. Simultanément, on encourage la sortie progressive de l'agriculture de toutes les exploitations agricoles qui ne sont pas "viables" économiquement, par des incitations à cesser leur activité et à céder leurs terres à des fermes souhaitant s'agrandir. Malgré un discours syndical alarmiste sur l'encouragement explicite à la diminution de l'effectif des exploitations agricoles, cette politique répond aux attentes de nombreux agriculteurs souhaitant agrandir leur exploitation plutôt que l'installation d'un maximum d'agriculteurs, mot d'ordre syndical largement partagé.

On est clairement dans une politique volontariste de structuration d'EAF de taille moyenne, par accroissement de taille et de capital investi, consensus clé de la cogestion État/Organisations professionnelles majoritaires. On ne prend simplement pas en compte le fait que (i) cette politique de structuration très sélective oblitère les logiques d'agrandissement provenant du soutien des prix qui profite aux exploitations qui sont déjà les plus grandes et que (ii) certaines petites exploitations que l'on voudrait voir disparaître vont en partie persister, simplement parce que leur faible capitalisation va aussi les protéger des dérives des aléas de prix du marché, des coûts financiers liés à l'investissement, en utilisant du matériel et des bâtiments déjà amortis.

Deux faits sociopolitiques saillants marquent la fin cette période : la procédure "États généraux du développement agricole " portée par É. Cresson, ministre de l'Agriculture en 1981-1983 et la mise en place des quotas laitiers en 1984 alors que M. Rocard lui a succédé en 1983.

Sous l'impulsion de É. Cresson et de son cabinet, s'engage un grand débat national et local sur les modèles de développement agricole et la recherche de politiques alternatives auquel participe une partie du monde professionnel agricole en alliance avec des " intellectuels » de la recherche engagés dans l'action politique (en particulier des chercheurs de l'Inra : C. Béranger, F. Colson, M. Gervais, P. Coulomb et $\mathrm{H}$. Nallet). Ce débat répond aux inquiétudes découlant de l'application des politiques de réforme de l'agriculture, adoptées par l'Europe et les États, qui se révèlent très sélectives. On s'interroge déjà sur les possibilités de sauvegarder l'emploi agricole tout en privilégiant un développement plus autonome des exploitations agricoles.

Avec M. Rocard, on revient à des ambitions plus modestes, et c'est une gestion interprofessionnelle de la cogestion à trois (État, Union européenne, secteur laitier) de l'offre agricole d'un secteur qui se met en place, avec la perspective d'un rôle fort donné aux interprofessions, et de fait une vision beaucoup moins réformiste, mais plus en adéquation avec une Europe plus soucieuse de résorber ses excédents que de réformer ses manières de produire.

11. Dispositif d'appui sélectif aux exploitations agricoles, mis en place dans le cadre de directives adoptées à Bruxelles, en mars 1972. 


\section{Les économistes ruraux et la défense du modèle de l'EAF}

Les années 1960-1970, période de transition de l'économie et de la politique agricole est aussi une période de riches développements sur la question de l'EAF, noyau intangible de l'agriculture française ou forme transitoire vers une concentration capitaliste dans de grandes exploitations. Ceci est bien évidemment accentué par la période de mai 1968 et le caractère engagé de beaucoup de travaux d'économie de cette époque.

Un point de repère central dans le questionnement de cette période est la conscience du changement de statut et de métier des paysans, qui se transforment en agriculteurs. Deux ouvrages de référence : La fin des paysans (Mendras, 1967), Une France sans paysans (Gervais, Servolin et Weil, 1965) prennent acte de la disparition des paysans et s'interrogent sur les catégories qui vont les remplacer, en reposant la question du mode de production (au sens marxiste du terme) vers lequel va évoluer majoritairement l'agriculture.

À l'Inra, dans le département d'Économie et sociologie rurale, schématiquement quatre interprétations s'opposent sur l'avenir de l'agriculture française. Certains économistes développent des travaux en microéconomie et en économie de la production dans une approche néomarginaliste. D. Bergmann, J.-M. Boussard, M. Petit sont parmi les représentants de ce courant. Les travaux de J.-M. Boussard (1987), en particulier, font une place importante à la modélisation en renouvelant l'usage de la programmation linéaire comme outil de représentation du fonctionnement des exploitations agricoles, dans une approche positive qui tranche avec l'approche normative qui accompagnait jusque-là les travaux menés dans ce cadre. Il met notamment en avant le rôle du risque dans les décisions des producteurs et développe des analyses et des travaux de modélisation qui permettent d'analyser les politiques agricoles sous un angle nouveau.

D'autres économistes se posent des questions sur l'évolution de l'ensemble des exploitations : C. Servolin, M. Gervais, H. Nallet et P. Coulomb (1974) proposent une lecture marxiste de l'évolution des exploitations. C. Servolin, montre comment la politique agricole est à la fois la condition incontournable de la reproduction de cette agriculture familiale modernisée, mais aussi l'instrument de sa différenciation. Enfin, comme le rappelle P. Coulomb, l'existence d'un foncier coûteux et limité, ainsi que l'existence de normes de rémunération minimale du travail interdisent l'extension généralisée des exploitations de type capitaliste dans la production agricole européenne, à l'exception de zones où la concentration foncière s'est constituée il y a très longtemps, comme par exemple dans les latifundia ibériques.

En opposition à cette interprétation, d'autres courants marxistes affirment que les progrès de l'ensemble de l'agriculture familiale proposés par la politique agricole sont très partiels car ils ne contrarient pas l'inévitable " absorption des paysans par l'accumulation capitaliste " se réalisant par l'intégration contractuelle des producteurs par les firmes agroindustrielles, privées ou coopératives ${ }^{12}$.

12. Des coopératives comme la CANA à l'époque (aujourd'hui Terrena) ont vécu ces évolutions conflictuelles, partagées entre l'efficacité de l'entreprise coopérative et la résistance des agriculteurs « intégrés » dans les productions avicoles, à la perte de leur identité. 
Enfin, L. Malassis (1979) conjugue une analyse de l'économie des exploitations agricoles à un diagnostic plus global du système alimentaire. Il montre que si les firmes d'amont et d'aval détiennent la plus grande part du pouvoir d'orienter la production au sein des exploitations agricoles, par des contrats d'intégration, la spécificité de "l'exploitation agricole néoartisanale " subsiste. Cette exploitation familiale garde un pouvoir d'initiative important dans sa capacité à progresser dans sa compétence technique, gestionnaire et patrimoniale. Cette catégorisation de l'exploitation qualifiée de néoartisanale entre la référence à l'artisan maittre de son appareil de production, de ses manières de faire et l'éleveur breton totalement intégré dans une firme agroalimentaire, représente un compromis commode pour parler de l'agriculteur et de l'EAF, sans trop s'engager sur l'évolution à long terme de ces formes de productions.

Enfin, notons que les économistes de l'Inra, à partir des années 1980, se consacrent beaucoup plus à un développement de l'économétrie et des modèles généraux de représentation de l'agriculture pour la planification, à l'échelle nationale, puis européenne, et réalisent beaucoup moins d'investigations directes sur l'économie des exploitations agricoles, considérée comme un domaine voué à un faible avenir scientifique. Les travaux réalisés en économie de la production se consacrent aux aspects méthodologiques de traitement de données, à mesure que les statistiques agricoles se développent. Le réseau d'information comptable agricole le (RICA), puis les typologies par orientation technico-économiques d'exploitation, OTEX, deviennent opératoires à partir du début des années 1970 (Butault, 1985). Malheureusement, les matériaux collectés par les statistiques publiques et leur traitement ne permettent guère d'apporter de contribution à la question entreprise/exploitation familiale, puisqu'ils ne comportent aucune information de type bilan, permettant de mesurer la concentration du capital. Simultanément, les différents courants de pensée marxistes s'épuisent en luttes internes, avec une montée en puissance de la pensée néolibérale, disqualifiant pour beaucoup de chercheurs tout ce qui ne repose pas sur de la quantification.

Les travaux du département Sad, à ses débuts, porteront majoritairement sur les zones et les systèmes de production animale, du fait des origines institutionnelles de ses fondateurs, mais aussi peut-être de la conscience du très grand écart entre une science zootechnique élaborée en laboratoire et station expérimentale, et les pratiques des éleveurs dans les zones d'agropastoralisme. Cette situation est différente de celle de la grande culture du Bassin parisien, où les retombées de la science agronomique végétale sont plus faciles à utiliser (génétique végétale et fertilisation). Cependant, le concept de «fonctionnement des exploitations agricoles " est d'abord une formulation d'agronome (Duru et al., 1988). Progressivement, il sera adopté par beaucoup de chercheurs du Sad (et par moi-même), quelle que soit leur discipline scientifique. Ces chercheurs partagent l'hypothèse que l'analyse du fonctionnement technique des systèmes de production est un élément décisif de compréhension des logiques de gestion des agriculteurs, combinant la poursuite d'objectifs et de stratégies plus globales sur leurs exploitations. L'élaboration de représentations du fonctionnement de l'exploitation est réalisée par l'observation des pratiques, l'analyse des résultats obtenus, et intègre aussi l'interprétation personnelle de l'agriculteur. 
C'est dans cette tension entre la recherche d'une plus grande productivité (prescrite par les économistes et les politiques publiques) et l'analyse des rationalités paysannes que va se fonder progressivement la nécessité d'une approche plus intégrée et systémique de l'exploitation agricole (Osty, 1978), dans ses dimensions d'échelle d'action de l'agriculteur hybridant ses pratiques avec des techniques prescrites par des instances externes, le plus souvent professionnelles.

On comprend bien aussi que cette posture de chercheurs, intégrateurs d'approches disciplinaires diverses, en réaction à une science descendante appliquée à la réalité, va trouver un écho très favorable, dans le monde de l'agriculture familiale, tel qu'il est porté à l'époque.

Les travaux des agronomes parisiens font un peu exception à cette spécialisation sur l'EAF, lorsqu'ils s'investissent dans le champ de l'organisation du travail dans des exploitations de grande culture avec des économistes de la gestion du laboratoire de Grignon (Attonaty et al., 1987).

La référence à Simon (1947), portée par Lemoigne, professeur d'économie à Aix-enProvence, avec le grand débat rationalité limitée/rationalité optimale servira assez largement de cadre de référence aux chercheurs du Sad, agronomes, zootechniciens, économistes pour comprendre les procédures de choix de gestion des agriculteurs et de leurs pratiques, qu'ils observent dans les exploitations. Ce faisant, l'écart avec un objectif de compréhension d'une dynamique d'ensemble de l'évolution des exploitations s'accroît dans cette perspective de positionnement dans la rationalité limitée d'acteurs concernés par une question déclinée à l'échelle individuelle ou locale. Cela vaut aussi pour les travaux sur l'économie et la gestion de l'exploitation agricole, recentrés progressivement vers l'agriculteur, ses objectifs, sa stratégie, etc. (Brossier et Chia, 1983 ; Brossier et al., 1997).

D’une certaine manière, la thèse que je réaliserai plus tard (1990-1995) remobilisera ce type d'approche combinant investigations agronomiques et économiques pour expliquer la décision de production dans différents types d'exploitations (Pluvinage, 1995), ainsi que la modélisation pour investiguer des points critiques du fonctionnement des exploitations (Jacquet et Pluvinage, 1997). Mais j'en tirerai aussi des leçons pour l'orientation de la politique agricole algérienne face au raisonnement de paysans confrontés à des aléas, ma situation de travail étant clairement représentative de l'agriculture des zones semi-arides algériennes. J'ai renoué ainsi le lien avec l'analyse des EAF dans les problématiques macroéconomiques classiques de l'économie agricole (Boussard, 1987).

Dans cette thèse, j'ai combiné des approches qui renvoient à l'analyse des pratiques de gestion des exploitations qui me conduisent aussi à des analyses beaucoup plus globales du développement agricole de l'Algérie, et de sa politique agricole. Mais je me suis aussi appuyé sur les travaux des agronomes des zones semi-arides, disciples de M. Sebillotte, ou des zones tropicales (Milleville, 1990), en convergence avec eux sur l'interprétation du sens des pratiques agricoles paysannes face aux aléas climatiques, pouvant apparaître comme contradictoires avec les prescriptions de la science agronomique "descendante " prônée sur les mêmes zones par les institutions officielles algériennes. Pour les chercheurs de ces institutions, le progrès de 
la production céréalière passait par l'adoption de variétés à paille courte, moins rustiques et produisant de fait moins de paille, et souvent moins appétentes pour le bétail, alors que pour les paysans de systèmes de polyculture élevage maghrébins, la paille est par excellence la ressource fourragère permettant de faire face aux aléas climatiques.

J'ai eu la chance de réaliser ce travail à un moment charnière où coexistaient, en Algérie, les exploitations individuelles, cœur de ma thèse, et les exploitations socialistes, peu de temps avant leur dissolution, qui relevaient pour une grande part de leur gestion, de la conduite de grandes entreprises, même si la planification socialiste y introduisait des contraintes spécifiques. Cela me permit de constater les difficultés de telles entreprises, malgré le fait de disposer d'un salariat parfois très compétent et un fort encadrement technique, à réagir à temps à tous les aléas du climat de ces régions et aussi aux prix irréguliers contingents des écarts de production.

Dans ce contexte de grands aléas, les EAF de par leur souplesse dans la gestion quotidienne, et moins bien dotées en capital que les domaines socialistes, restent un cadre économique bien plus performant pour gérer les risques climatiques et économiques.

\section{Depuis 1992, conséquences de l'évolution de la PAC sur le débat exploitation agricole familiale, vs. entreprise avec salariés}

La première réforme de la PAC en 1992, puis celle de 2003, changent radicalement le contexte de la production agricole européenne : la dérégulation des marchés agricoles face à la mondialisation et aux exigences de l'Organisation mondiale du commerce, les crises sectorielles et budgétaires, l'élargissement de l'UE, reposent la question d'un modèle unique de développement de l'agriculture européenne du type de celui des années 1960. Les progrès de revenus espérés ne peuvent plus être globalement attendus de progrès de rendements qui stagnent. Les cultures et élevages intensifs génèrent des externalités négatives quand on augmente les intrants et les chargements animaux.

Devant cette situation difficile, la tension entre la recherche d'une toujours plus grande productivité du travail et l'émergence de nouvelles finalités de l'agriculture s'accroît, conduisant à un écart de plus en plus marqué entre exploitations agricoles se projetant en permanence dans des logiques d'agrandissement des superficies cultivées, avec des systèmes de production les plus simplifiés possibles, et celles innovant dans la recherche d'adaptation à des contextes locaux.

Le nombre d'exploitations continue à diminuer, les plus grandes augmentant en effectif et en taille plus rapidement que dans la période antérieure à 1990 . Globalement, les exploitations agricoles réemploient un peu plus de salariés à partir de 1995 (Rattin, 1999). On assiste aujourd'hui à une croissance de la place des salariés, dans la main-d'œuvre agricole, qui réalisent $33 \%$ du travail en 2011, contre 
$28 \%$ en 2000 (Tab. 1). Cependant, cette évolution semble plus correspondre à des emplois partagés entre plusieurs exploitations qu’à un changement radical de la nature de l'exploitation agricole.

Tab 1. Évolution de la place des salariés dans l'exploitation agricole (source Agreste).

\begin{tabular}{|lccc}
\hline Effectif (x 1000 UTA) & $\mathbf{1 9 8 8}$ & $\mathbf{2 0 0 0}$ & $\mathbf{2 0 1 1}$ \\
\hline Total salariés agricoles & 234 & 231 & 211 \\
\hline - Dont salariés permanents & 156 & 140 & 141 \\
\hline Main-d'œuvre familiale & 883 & 584 & 432 \\
\hline MO sal./MO Totale & $21 \%$ & $28 \%$ & $33 \%$ \\
\hline
\end{tabular}

La question du salariat comme force de travail principale dans une typologie des exploitations agricoles selon leur nature, n'est cependant pas décisive, tout au moins en France où les institutions (politiques foncières et politiques d'accès à l'installation) et normes sociales, jouent encore un rôle majeur dans l'organisation sociale de l'agriculture. Elle n'est centrale que dans les situations particulières d'innovations radicales dans le système productif qui peuvent bouleverser les pratiques productives de l'exploitation ${ }^{13}$.

Dans d'autres pays, où les normes sociales et syndicales sont beaucoup moins favorables aux salariés, de grandes exploitations industrielles (en croissance forte dans des activités agroexportatrices : fruits et légumes, horticulture, élevage hors-sol et élevage bovin extensif) se développent brutalement au détriment des petites et moyennes exploitations. Ce développement se fait, par ailleurs, avec peu de préoccupations de l'impact écologique des productions mises en œuvre ou de la santé des salariés.

À l'opposé de ce mouvement d'agrandissement des exploitations avec une accentuation de la simplification des systèmes de production, on a assisté durant les années 1999-2002, à une tentative de modification fondamentale de la politique agricole française de financement public des exploitations agricoles. Cette réorientation engagée au nom d'un financement de la multifonctionnalité de l'agriculture, combinant trois finalités, économiques environnementales et sociales, s'est traduite concrètement par la mise en place des contrats territoriaux d'exploitations (CTE). Ces contrats permettaient d'intégrer les stratégies d'exploitation à des finalités territoriales rassemblant, à l'échelle locale, différents acteurs agricoles et non agricoles (avec des objectifs de protection de la biodiversité, de la sauvegarde de paysages, etc.).

Pour plein de raisons, tant liées aux difficultés d'articulation à la politique agricole européenne, qu'aux réticences de beaucoup d'organisations professionnelles agricoles, et à la brièveté de l'expérience écourtée par le changement politique de 2002,

13. Voir l'exemple des transformations de la culture du melon (Pluvinage, 2011). 
cette réorientation n'a pas eu le temps de transformer globalement le paysage agricole, et surtout les manières de penser des agriculteurs sur le développement de leur exploitation dans l'avenir.

Pourtant les " petites fermes » (expression de la Confédération paysanne) restent nombreuses, même si elles diminuent statistiquement plus rapidement que les autres (Desriers, 2007). On verra, au travers des travaux des chercheurs, qu'elles s'appuient beaucoup sur la pluriactivité des ménages et sur des motivations très diverses, variables selon les régions, les contextes territoriaux et les systèmes de production.

\section{Nouvelles investigations des économistes ruraux sur la diversité des formes de production en agriculture et la diversité des exploitations}

Depuis les années 1990, quelques économistes (et sociologues) renouvellent la construction des typologies d'exploitations en critiquant les approches uniquement centrées sur les exploitations dites " professionnelles ". Il s'agit d'intégrer à l'analyse du secteur agricole d'autres acteurs, tels que des ménages exerçant, pour diverses raisons (économiques et choix de vie), une activité agricole à titre secondaire. Cette réflexion conduit à s'intéresser à des types d'exploitations le plus souvent "ignorées " par la profession et le ministère de l'Agriculture. C. Laurent, P. Mundler, D. Ponchelet et S. Bellon (1998), notamment, poursuivront ainsi des travaux conduits dans les années 1960 par A. Brun, P. Lacombe et C. Laurent (1982) sur la pluriactivité. Ces travaux aboutiront ultérieurement (Laurent et Rémy, 2006) à une remise en cause plus radicale du concept d'exploitation agricole, celle-ci devant être plus perçue à leurs yeux comme l'assemblage des activités des membres d'un ménage faisant système, que comme une stricte unité de production assemblant parfois des activités et revenus non agricoles avec celles relevant de la production agricole. Ceci renvoie aussi à toute la question des conséquences de l'individuation en agriculture, traitée par ailleurs dans cet ouvrage.

Personnellement, durant cette période de tentative française d'une réforme $\mathrm{du}$ financement public de l'agriculture autour du contrat territorial d'exploitation (CTE), je me suis investi dans l'analyse des conditions d'une meilleure reconnaissance publique de la multifonctionnalité des exploitations. J'ai pu observer que, dans certaines situations, l'intégration des préoccupations territoriales dans les projets des agriculteurs a été largement discutée avec des acteurs non agricoles et a abouti, au moins dans les dimensions agrienvironnementales, à des modifications non négligeables de la manière d'envisager des changements de pratiques agricoles dans la Drôme, dans le Diois et dans le nord de l'Ardèche (Pluvinage et Tchakérian, 2005), c'est-à-dire dans des régions de petites EAF.

Des comparaisons menées avec d'autres chercheurs en Rhône-Alpes nous ont permis d'analyser les facteurs d'adhésion à ces évolutions dans les territoires fragilisés, soit par la menace de désertification, soit par l'urbanisation des territoires périphériques 
des villes, où le maintien de l'agriculture passe par un mouvement de défense plus général d'espaces agricoles, défense qui n'est possible que dans des procédures d'alliance ou de protection avec des collectivités territoriales (Bernard et al., 2006).

Globalement, j'ai pu constater que la trajectoire sociale de l'agriculteur et de sa famille, combinée à des données structurelles de son exploitation, était un facteur décisif de ce nouveau positionnement social et économique d'agriculteurs (Pluvinage, 2009), en interaction avec diverses dynamiques territoriales. Nous avons tenté de cerner plus précisément les critères de rentrée dans ce jeu territorial local ou régional des logiques de fonctionnement d'exploitations, et la convergence ou les divergences de ces dynamiques avec des stratégies de filières (Praly et al., 2010, Vandenbroucke, 2013), comme nous en témoignons dans un autre chapitre de cet ouvrage. Les logiques de ces petites EAF sont variées avec, cependant, fréquemment, une finalité patrimoniale vécue autant comme un souhait personnel que comme un nouveau positionnement économique comportant des opportunités marchandes en devenir.

\section{Conclusion générale}

Que conclure de ce parcours au travers de cinquante années d'investigations sur la nature de l'opposition EAF/EA, soulevée au début de ce chapitre.

Une première ligne de séparation entre la conception de la production agricole comme une activité économique d'entreprise, et une autre : celle d'une activité aux facettes multiples, structurée entre autres par le caractère familial du travail des capitaux et du foncier, constitue une clé de lecture déterminante de la compréhension des dynamiques du système productif agricole. Les objectifs de l'activité productive et les systèmes de valeur sous-jacents produisent une ligne de partage, qui recoupe souvent celle inhérente aux caractères familiaux de l'exploitation, mais mobilise aussi d'autres dimensions dans l'analyse.

Dans l'exploitation agricole paysanne de Tchayanov (1990), comme dans celle de la Confédération paysanne, de l'agriculture diversifiée des réseaux d'agriculture durable (RAD) ou encore dans la plupart des exploitations en agriculture biologique en France, on se situe dans des projets de production qui se déroulent sur le temps long et que l'on ne va pas évaluer uniquement en termes de revenu et de productivité immédiate, mais en référence à une autre rationalité plus globale, qui se décline sur le long terme. Toutes nos enquêtes montrent combien nombre d'agriculteurs sont capables de sacrifier un revenu disponible annuel au profit d'un revenu à venir en partie aléatoire du fait d'inconnues marchandes ou de connaissances incertaines sur les itinéraires techniques adéquats. Ils le font sur la base de cheminements divers, qui renvoient souvent à une conception du métier qui leur semble plus conforme à des idéaux, des expériences, et des résultats qu'ils jugent possibles d'obtenir, tout en restant cohérents avec leurs objectifs personnels. 
On continue donc à observer en agriculture deux conceptions économiques différentes :

- certaines exploitations se réferent à des idéaux d'optimisation économique, où l'accroissement de taille, la spécialisation, associée au développement du salariat, visent un accroissement rapide du revenu et les moyens d'une capitalisation infinie, même si celle-ci n'est qu' exceptionnellement possible en France ;

- d'autres se réferent à un souci de durabilité économique, environnementale et sociale qui les incite à rechercher des voies de consolidation technique, économique et sociale, assurant un revenu suffisant plutôt que maximum, privilégiant plus la viabilité en référence à de nombreux critères que la seule performance technico-économique.

Cette opposition conceptuelle de deux manières de considérer le métier d'agriculteur et l'exploitation ou l'entreprise n'est pas nouvelle ; ce qui est nouveau c'est l'accroissement du contraste, qu'expriment par exemple les montants des quantités de facteurs de production et de superficie mobilisées ${ }^{14}$, ainsi que dans une certaine mesure, les rapports aux différents acteurs de la société, même si les dimensions sociales et culturelles propres à chaque région et à la diversité des systèmes de production, donnent une dimension différente à ces évolutions.

Une seconde conclusion se dégage de notre analyse chronologique au-delà des invariants du débat EAF/EA, c'est celle d'un ciblage variable dans le temps de la politique agricole sur l'une ou l'autre de ces formes sociales de production, et particulièrement sur ce que l'on imagine comme paradigme moteur du progrès à l'époque considérée.

La première période fait, en quelque sorte, feu de tout bois. Il faut produire plus et peu importe le modèle de structure de production; les incitations financières à l'équipement individuel et collectif, combinées au soutien des marchés, sont censées donner les moyens à toutes les exploitations d'assurer leur revenu et leur avenir.

La seconde serait, en quelque sorte, un âge d'or de l'EAF, objet central de l'action publique, compromis social « idéal " pour gérer contraintes économiques et progrès scientifiques appliqués à l'agriculture, délaissant apparemment le débat entre des avantages et inconvénients de la grande entreprise. La politique agricole met en place tant au niveau français qu'européen, des dispositifs d'incitation visant une plus grande convergence des structures, vers les exploitations de taille moyenne ; cependant dans cette ambiance de modernisation des exploitations familiales de la «classe moyenne ", la politique agricole ne prend pas en compte (ou ignore) la réalité de la dynamique marchande, élément encore plus décisif de différenciation des structures que l'action publique, particulièrement dans les systèmes de grande culture qui sont ceux qui bénéficient le plus des moyens financiers de la PAC.

La troisième période, sans abandonner le discours sur l'exploitation agricole familiale, ciment du vote professionnel et politique agricole, remet en valeur la terminologie de l'entreprise et la nécessité de changements structurels plus radicaux pour

14. Voir les controverses autour de la mise en place d'une ferme de 1000 vaches aux environs d'Amiens. 
faire face à des obligations de concurrence internationale ou aux réticences sociales découlant des astreintes quotidiennes de la production artisanale (en production laitière par exemple). Le désengagement progressif de l'intervention publique, comme les encouragements du monde agroindustriel qui souhaite la concentration de ses approvisionnements, contribuent à l'affirmation de cette tendance.

Cependant, de nombreuses expressions de résistance s'expriment en permanence contre cette dynamique, ressentie comme destructrice des EAF, et du métier d'agriculteur " néoartisanal » de L. Malassis. Ces résistances sont autant syndicales (Confédération paysanne, MODEF, mais aussi pour partie de la Coordination rurale) que productives intégrant une forte dimension écologique (FNAB, FNCIVAM, RAD, etc. ${ }^{15}$. Elles incarnent beaucoup d'initiatives alternatives dans la production agricole, sans qu'un consensus politique aboutisse à remettre en cause plus radicalement la dynamique capitalistique du couple industrialisation-agrandissement, et les ambiguïtés des politiques agricoles.

Les perspectives de l'accroissement de cette bipolarité entre entreprise agricole et exploitation agricole familiale sont difficiles à préciser, au-delà des facteurs classiquement favorables à l'agrandissement des fermes, déjà soulignés, comme l'absence de régulation foncière ou salariale. Se combine à cela la déclinaison de nouveaux progrès techniques décisifs en termes de productivité du travail, tout en exigeant des capitaux importants.

Par exemple, la dynamique de l'évolution en cours et à venir des exploitations laitières françaises dans un contexte de disparition de quotas et de progrès techniques fortement discontinus (liés à l'adoption des robots de traite, la séparation des exploitations laitières entre celles qui se consacrent à la production de fourrages et celles de taille très importante se consacrant uniquement à l'élevage) pourrait être un terrain de lecture assez illustratif de l'avenir de l'EAF, entre résistance de petites exploitations laitières, certaines diversifiant leur système de production et grandes étables de plusieurs centaines de vaches.

Dans les systèmes spécialisés comme les cultures légumières de plein champ, la mécanisation des opérations culturales, et de récolte, contribue aussi au développement de grandes exploitations spécialisées et avec des salariés, comme dans le département des Landes par exemple.

À l'inverse, dans d'autres productions comme les productions fruitières, ces dynamiques de mécanisation apparaissent beaucoup plus difficiles à mettre en œuvre, et freinent le développement d'entreprises spécialisées.

C'est donc une lecture par grands systèmes et filières de production et régions, en tenant compte, d'une part, d'une vision prospective des innovations à venir, d'autre part, des rapports ville/campagne spécifiques aux différentes régions, qu'il faudrait réaliser pour faire un point plus précis sur les évolutions à venir du débat exploitation agricole néo-artisanale/entreprise agricole avec salariés.

15. Fédération nationale d'agriculture biologique, Fédération nationale des centres d'initiatives pour valoriser l'agriculture et le milieu rural, Réseau agriculture durable. 


\section{Bibliographie}

Attonaty, I.M., Laporte, C., Papy, F., Soler, L.G., 1987. La simulation de l'organisation du travail comme outil de gestion de l'exploitation agricole. Application à la grande culture, Études et Recherches sur les Systèmes Agraires et le Développement, 10, 1-48.

Bernard, C., Dobromez, L., Pluvinage, J., Dufour, A., Havet, A., Mauz, I., Pauthenet, Y., Rémy, J., Tchakérian, E., 2006. La multifonctionnalité à l'épreuve du local : les exploitations agricoles face aux enjeux des filières et des territoires, Cahiers Agricultures, 15, 6, 523-528.

Boussard, J.-M., 1987. Économie agricole, Paris, Economica.

Brossier, J., Chia, E., 1983. Connaissance des outils de gestion effectivement utilisés par les agriculteurs et amélioration de leur pertinence. Les pratiques de trésorerie. Document ANDA.

Brossier, J., Chia, E., Marshall, E., Petit, M., 1997. Gestion de l'exploitation agricole familiale Eléments théoriques et méthodologiques, ENESAD-CNERTA.

Brun, A., Lacombe, Ph., Laurent, C., 1982. La pluriactivité des agriculteurs, hommes, espaces, politiques, INRA, Série Économie et sociologie rurales.

Butault, J.-P., 1985. La nomenclature OTEX/CEDEXE, intérêts et limites in " Systèmes de production et transformations de l'agriculture, INRA Paris, Tome 2, 51-56.

Chombart de Lauwe, J., Poitevin, J., Tirel, JC., 1963. Nouvelle gestion des exploitations agricoles, Paris, Dunod.

Desriers, M., 2007. L'agriculture française depuis cinquante ans : des petites exploitations familiales aux droits à paiement unique, INSEE, Agriculture, nouveaux défis, 17-30

Dumont, R. ,1951. Voyages en France d'un agronome, Paris, Ed. Génin.

Duru, M, Papy, F., Soler, L.G., 1988. Le concept de modèle général et l'analyse du fonctionnement de l'exploitation agricole, Comptes-rendus de l'Académie d'agriculture, 74, 4, 94-96.

Eldin, M., Milleville, P., 1989. Ouvrage collectif sur le risque en agriculture, Paris, Orstom.

Hervieu, B., Purseigle, F., 2011. Des agricultures avec des agriculteurs, une nécessité pour l'Europe, Projet n 321.

Gervais, M., Servolin, C., Weil J., 1965. Une France sans paysans, Paris, éditions du Seuil.

Jacquet, F., Pluvinage, J., 1997. Climatic uncertainty and farm policy : A discrete stochastic programming model for cereal-livestock farms in Algeria, Agricultural systems, 53, 4, 387-407.

Laurent, C., Cartier, C., Fabre, C., Mundler, P., Ponchelet, D., Remy, J., 1998. L'activité agricole des ménages ruraux et la cohésion économique et sociale, Économie Rurale, 244, 12-21.

Laurent, C., Remy, J., 2000. L'exploitation agricole en perspective, Le courrier de l'environnement, 141, 5-23. 
Malassis, L., 1979. Économie de la production et de la consommation agro-alimentaire, Paris, Cujas.

Mendras, H., 1967. La fin des paysans, Paris, SÉDÉIS.

Neveu, A., 2010. Le modèle européen d'exploitation agricole moyenne a-t-il encore un avenir ? Comptes-rendus de l'Académie d'agriculture de France, 96, 2.

Osty, P.-L., 1978. L'exploitation agricole vue comme un système, BTI, 326, 43-49.

Pluvinage, J., 1995. Les systèmes de production céréales élevage et la gestion du risque dans les plaines sèches méditerranéennes, thèse sciences économiques Montpellier.

Pluvinage, J., 2009. Multifonctionnalité, stratégies individuelles d'agriculteurs, et dynamiques territoriales spécifiques en Rhône-Alpes in Nouvelles questions agraires, Exploitants, fonctions et territoires, Cornu P. et Mayaud J.L. [Ed], Sèvres, Boutique de l'Histoire, 249-275.

Pluvinage, J., 2011. Innovations, alternatives, et diversité des exploitations agricoles, Pour, 212, 187-197.

Pluvinage, J., Tchakérian, E., 2005. Quelles relations entre les formes de multifonctionnalité des exploitations agricoles et la configuration du développement territorial? (Préalpes drômoises et nord Ardèche), Rapport de synthèse, programme PSDR Rhône-Alpes, INRA Montpellier.

Praly, C., Chazoule, C., Delfosse, C., Pluvinage, J., 2007. SYAL et relocalisation des agricultures européennes. Une application à l'arboriculture de la Moyenne Vallée du Rhône, Économies et Sociétés, Série Systèmes agroalimentaires, 29, $1547-1565$.

Rattin, S., 1999. Salariés agricoles permanents : entre 1995 et 1997, 4000 emplois permanents supplémentaires, Agreste cahiers, 37, 15-21.

Servolin, C., Gervais, M., Nallet, H., Coulomb, P., 1974. L'agriculture dans le système social-recueil d'articles, Paris, INRA.

Simon, H., 1947. Administrative Behavior, New York, Macmillan.

Tchayanov, A., 1990. L'organisation de l'économie paysanne, Paris, Librairie du Regard (traduction du russe de l'ouvrage de 1924).

Vandenbroucke, P., 2013. Transformation de l'unité de production agricole, d'une exploitation sectorielle à une exploitation agricole territoriale. Exploitations agricoles, agriculteurs et territoires dans les Monts du Lyonnais et en Flandre intérieure de 1970 à 2010, Thèse de Géographie, Aménagement et Urbanisme. Dir. Pluvinage J., Laboratoire d'études rurales, Université Lyon 2, Lyon. 